\title{
Defect Kinetics and Resistance to Amorphization in Zirconium Carbide
}

\author{
Ming-Jie Zheng ${ }^{\mathrm{a}, \mathrm{b}}$, Izabela Szlufarska ${ }^{\mathrm{a}, \mathrm{c}}$, Dane Morgan ${ }^{\mathrm{a}, \mathrm{c}, \text { * }}$ \\ ${ }^{a}$ Department of Materials Science and Engineering, University of Wisconsin-Madison \\ ${ }^{b}$ Institute of Nuclear Energy Safety Technology, Chinese Academy of Sciences, China \\ ${ }^{c}$ Materials Science Program, University of Wisconsin- Madison \\ *Emails: ddmorgan@wisc.edu, szlufarska@wisc.edu
}

\begin{abstract}
To better understand the radiation response of zirconium carbide $(\mathrm{ZrC})$, and in particular its excellent resistance to amorphization, we have used density functional theory methods to study the kinetics of point defects in $\mathrm{ZrC}$. The migration barriers and recombination barriers of the simple point defects are calculated using the ab initio molecular dynamics simulation and the nudged elastic band method. These barriers are used to estimate $\mathrm{C}$ and $\mathrm{Zr}$ interstitial and vacancy diffusion and Frenkel pair recombination rates. A significant barrier for $\mathrm{C}$ Frenkel pair recombination is found but it is shown that a large concentration of $\mathrm{C}$ vacancies reduces this barrier dramatically, allowing facile healing of radiation damage. The mechanisms underlying high resistance to amorphization of $\mathrm{ZrC}$ were analyzed from the perspectives of structural, thermodynamic, chemical and kinetic properties. This study provides insights into the amorphization resistance of $\mathrm{ZrC}$ as well as a foundation for understanding general radiation damage in this material.

Keywords: Zirconium carbide, Resistance to amorphization, Defect kinetics, Energy barriers
\end{abstract}

PACS: 61.72.Cc, 61.80.-x, 28.52.Fa, 82.20.Wt 


\section{Introduction}

Zirconium carbide $(\mathrm{ZrC})$ is a promising candidate for structural materials in high temperature gas cooled reactor (HTGCR) as compared to $\mathrm{SiC}$ due to its high melting temperature[1], superior resistance to irradiation damage[2-3] and possibly superior resistance to fission product attack and permeation[4]. It has been proposed for use as coating for deep-burn Tristructural-isotropic (TRISO) nuclear fuel particles both as an oxygen getterer and structural cladding $[3,5]$. By using $\mathrm{ZrC}$ in combination with, or in place of, $\mathrm{SiC}$, one may be able to reduce particle rupture, chemical degradation, release of fission product and enable increased gettering of oxygen[2]. The use of $\mathrm{ZrC}$ in TRISO fuel or other nuclear applications requires a thorough understanding of its irradiation responses. However, the radiation effects on $\mathrm{ZrC}$ are still poorly understood.

$\mathrm{ZrC}$ has a rock-salt crystal structure, consisting in two interpenetrating sublattices of $\mathrm{Zr}$ and $\mathrm{C}[6]$. As a transition metal carbide, $\mathrm{ZrC}$ has an unusual combination of physical properties[7]. In the one hand, it has high melting temperature and hardness[6-7]. On the other hand, it has good electric and thermal conductivities, like metals[6]. There exists a wide range of nonstoichiometric compositions $\operatorname{ZrC}_{x}(0.6<x<1.0)$ [8] over which the lattice structure remains unchanged. The strong covalent $\mathrm{Zr}-\mathrm{C}$ bonding confers its mechanical properties[7] and induce its brittleness below the brittle-ductile transition temperature of $1200^{\circ} \mathrm{C}[9]$. Such brittleness of $\mathrm{ZrC}$ makes its manufacture difficult and hinders its application. The hardness of $\mathrm{ZrC}$ decreases with increasing temperature[6] and it becomes significantly softer at high temperature. 
The existing experimental literature data on $\mathrm{ZrC}$ irradiation effects is quite limited[10]. Gosset et al. irradiated $\mathrm{ZrC}$ with $4 \mathrm{MeV} \mathrm{Au}$ ion at room temperature and monitored the microstructural evolution through Grazing Incidence X-ray diffraction (GIXRD) and transmission electron microscopy (TEM)[11]. They found a moderated swelling and saturated internal strains, which originates from the high density of small faulted dislocation loops. $\mathrm{ZrC}$ was irradiated with a $2.6 \mathrm{MeV}$ proton beam at $800^{\circ} \mathrm{C}$ by $\mathrm{Yang}$ et al.[12]. A high density of Frank loops dominates the irradiated microstructure and a slight lattice expansion was discovered. The hardness and fracture toughness were found to increase with increasing radiation dose. Snead et al. irradiated zone-refined $\mathrm{ZrC}$ with fast neutrons at the temperature range of $635-1480^{\circ} \mathrm{C}[3]$. The microstructural defects were dominated by dislocation loops, which underwent a gradual transition from Frank loops to prismatic loops above $1280^{\circ} \mathrm{C}$. Only modest changes were observed under irradiation in the mechanical properties of hardness, elastic modulus, and indentation fracture toughness, and the lattice parameter varied slightly $(<0.2 \%)$. Thus the $\mathrm{ZrC}_{0.93}$ was found to be quite stable under irradiation[3]. Up to now, no amorphization has been observed in $\mathrm{ZrC}[13]$, which is consistent with the generally stable properties observed under irradiation by Snead et al.[3] This resistance to amorphization (RTA) is not shared by some related carbides, e.g., $\mathrm{SiC}$, and the origin of this amorphization resistance may shed light on both the radiation response of $\mathrm{ZrC}$ and amorphization processes in general. This study therefore seeks to understand the basic point defect kinetic properties of $\mathrm{ZrC}$ as a starting point to understand its amorphization and more general irradiation response. 
Previous computational modeling of defect properties and irradiation effects in $\mathrm{ZrC}$ consists of just a few studies. Li et al. calculated the formation energy and migration barrier of C vacancy to develop an interatomic potential model[14]. Classical molecular dynamics (MD) simulation was used by Brutzel et al. to study the primary damage created by collision cascade in a $\mathrm{ZrC}$ matrix. No amorphization was observed in their cascades, and the point defects created included $\mathrm{C}$ ( $\mathrm{Zr}$ ) interstitials, a few $\mathrm{Zr}$ antisites, and clusters of two kinds of interstitials. A comprehensive ab initio study of point defect structures and energetics in $\mathrm{ZrC}$ was performed by $\mathrm{Kim}$ et al.[2] The most stable interstitial structure was determined to be the $\mathrm{C}$ interstitial (C-C-C trimer). $\mathrm{C}$ vacancies and interstitials were found to be much more stable than $\mathrm{Zr}$ vacancies and interstitials. Antisite defects were found to be quite unstable, suggesting chemical disorder will be low in $\mathrm{ZrC}$. Razumovskiy et al.[15] studied the migration of $\mathrm{Zr}$ via a vacancy-mediated mechanism in $\mathrm{TiC}$ and $\mathrm{ZrC}$. For $\mathrm{ZrC}$ they found that the $\mathrm{V}_{\mathrm{Zr}}-6 \mathrm{~V}_{\mathrm{C}}$ cluster was more stable than the isolated $\mathrm{V}_{\mathrm{Zr}}$ and that this species migrated with a barrier of $7.46 \mathrm{eV}$, the same value obtained from experiment[16]. The exact thermodynamic conditions under which this result was calculated ( $\mathrm{Zr}$ - or C- rich) are not clear to us from the paper but it appears to be a condition where $\mathrm{C}$ vacancies are readily available at no energy cost, which is consistent with less C-rich or irradiated conditions. We will discuss further the implications of these results when we consider $\mathrm{Zr}$ diffusion below. Jiang et al.[17] directly studied the amorphization process of both $\mathrm{ZrC}$ and $\mathrm{SiC}$ with ab initio molecular dynamics by adding C Frenkel Pairs (C FP) into both systems, annealing briefly, and tracking the long-range structural order. Jiang et al. found that $\mathrm{SiC}$ readily amorphized but $\mathrm{ZrC}$ did not. This difference was explained in terms of the intrinsic instability of the 
Si sublattice vs. the intrinsic stability of the $\mathrm{Zr}$ sublattice, where the latter protects the $\mathrm{ZrC}$ from amorphization under $\mathrm{C}$ disorder. Jiang et al. also showed that for $\mathrm{ZrC}$, no matter how many C FPs were added, the point defects did not accumulate to sufficient energy to destabilize the crystalline $\mathrm{ZrC}$ as compared to amorphous $\mathrm{ZrC}$. This result suggests that the approximately athermal annealing processes were accessible in $\mathrm{ZrC}$ at high point defect concentrations. As will be shown in this work, such a result is surprising given the barriers to point defect recombination for dilute defects, but seems to emerge due to defect interactions at high point defect density. While these previous studies provide a number of valuable insights, they do not provide a complete picture of point defect migration and recombination, so we undertake to provide such a picture in this work.

In this work, we use nudged elastic band (NEB) and ab initio molecular dynamics (AIMD) methods to calculate the migration and recombination barriers of point defects in ZrC. The mobility of each point defect is analyzed by calculating the diffusion coefficients of each type. The annealing properties of $\mathrm{ZrC}$ were studied by calculating the recombination barriers of isolated Frenkel pairs as well as in the presence of other defects. We then try to understand the fundamental mechanisms underlying the high RTA of $\mathrm{ZrC}$ by reviewing previous amorphization models and properties of $\mathrm{ZrC}$.

\section{Methods}


The $a b$ initio calculations were carried out using the Vienna ab initio simulation package (VASP) [18-21] based on the density functional theory (DFT) and the projectoraugmented-wave (PAW) method. The exchange-correlation was treated in the generalized gradient approximation (GGA), as parameterized by Perdew, Burke, and Ernzerhof (PBE)[22]. The PAW potentials were generated with the following valence electronic configurations: $5 s 4 d 5 p$ for $\mathrm{Zr}$ and $2 s 2 p 2$ for $\mathrm{C}$.

Two kinds of $\mathrm{ZrC}$ supercells and associated k-point meshes were used. All supercells are multiples of the cubic 8 -atom conventional rock-salt cell. A 64 -atom $2 \times 2 \times 2$ supercell with a converged $5 * 5 * 5 \mathrm{k}$-point mesh was used to study the diffusion of an interstitial or a vacancy, and a 216 -atom $3 \times 3 \times 3$ supercell with a converged $3 * 3 * 3 \mathrm{k}$-point mesh was used to study the recombination of Frenkel pairs. For the $\mathrm{Zr}$ FP recombination, $1 * 1 * 1 \mathrm{k}$ point mesh was used for the nudged elastic band (NEB)[23] calculation due to convergence problems with the $3 \times 3 \times 3 \mathrm{k}$-point mesh. While this lower k-point mesh is not very well converged for the total energy we found it gave acceptable errors for the migration energies. Specifically, for selected situations we checked the barrier calculated for the $1 * 1 * 1 \mathrm{k}$-point mesh compared to the barrier from a $3 * 3 * 3 \mathrm{k}$-point mesh and found the barrier difference is only about $0.05 \mathrm{eV}$. Thus the k-point mesh for NEB calculation is $3 * 3 * 3$ for $\mathrm{C}$ recombinations, and $1 * 1 * 1$ for $\mathrm{Zr}$ recombinations. All calculations used an energy cut-off of $600 \mathrm{eV}$. In each kind of supercell, the energy error on defect formation and migration energies is less than $1 \mathrm{meV} /$ atom with respect to the $\mathrm{k}$-point convergence and energy cut-off. 
The NEB method has been used to calculate the energy barriers for the migration of intrinsic defects and recombination of Frenkel pairs. In both cases, one of the key issues is to find the minimum energy path. To achieve this effectively, we use AIMD simulation as a guide for the path, following the approach used in Ref.[24]. These AIMD runs were performed at a temperature of $2800 \mathrm{~K}$ with a time step of 3 fs and lasted for $3 \sim 10 \mathrm{ps.}$ The AIMD runs with a smaller time step of $1 \mathrm{fs}$ were also checked, which suggested a similar path as that from the AIMD runs with longer time step of $3 \mathrm{fs}$.

As described in Ref. [24], the formation energy $\left(E_{f}\right)$ and the migration barrier $\left(E_{m}\right)$ are used as approximations for the free energies of formation $\left(F_{f}\right)$ and migration $\left(F_{m}\right)$, respectively. For the neutral state considered in $\mathrm{ZrC}$ (the metallic band structure means that no charged defects will form), the defect formation energy $E_{f}$ is given by [2]

$$
E_{f}=E_{\text {def }} \quad E_{\text {perfect }}+\sum_{i}^{\prime} n_{i} \text {, }
$$

where $E_{\text {def }}$ is the energy of the defected cell, $E_{\text {perfect }}$ is the energy of the perfect cell, $\Delta n_{i}$ is the change in the number of species $i(i=\mathrm{Zr}$ or $\mathrm{C})$ when the defect forms, and $\mu_{i}$ is the chemical potential of species $i$. We set ${ }_{i}={ }_{i}^{0}$ for species $i$-rich condition. In this work we focus on C-rich conditions, which is consistent with $x \approx 1$ in $\mathrm{ZrC}_{x}$. Formally one can treat $\mathrm{Zr}$-rich conditions with the transformation ${ }_{Z r}(\mathrm{Zr}$-rich $)={ }_{Z r}(\mathrm{C}$-rich $) \quad E_{Z r C}$ and ${ }_{C}(\mathrm{Zr}$-rich $)={ }_{C}(\mathrm{C}$-rich $)+E_{\mathrm{ZrC}}$, where $E_{\mathrm{ZrC}}={ }_{\mathrm{ZrC}}^{0} \quad{ }_{\mathrm{Zr}}^{0} \quad{ }_{\mathrm{C}}^{0}=-1.64 \mathrm{eV}$. However, under $\mathrm{Zr}$-rich conditions when the stoichiometry of $\mathrm{ZrC}_{x}$ is far from $x=1$ the formation energy values might be different from those in the stoichiometric $\operatorname{ZrC}_{x}(x=1)$ [15]. 


\section{Migration barriers and diffusion coefficients}

The mobility of intrinsic point defects plays an important role in the resistance to amorphization and general radiation response, since higher mobility generally enables more annealing in materials. Here we focus on the self-diffusion of intrinsic point defects in $\mathrm{ZrC}$, which include $\mathrm{C}$ interstitial $\left(\mathrm{C}_{\mathrm{i}}\right), \mathrm{C}$ vacancy $\left(\mathrm{V}_{\mathrm{C}}\right), \mathrm{Zr}$ interstitial $\left(\mathrm{Zr}_{\mathrm{i}}\right)$ and $\mathrm{Zr}$ vacancy $\left(\mathrm{V}_{\mathrm{Zr}}\right)$. To study the diffusivities of these point defects, we need to calculate their migration barriers, which require us to analyze the diffusion mechanisms and find the minimum energy diffusion path. The diffusion path of vacancies can be established based on the geometry and symmetry of the system. But the diffusion of interstitials is not so intuitive, thus we resorted to the AIMD simulation to guide us to find the possible paths. After careful analysis, the diffusion path extracted from AIMD run was divided into several basic hops. Then we calculated the migration barrier for each hop using NEB method, as listed in Table 1.

For the diffusion of $\mathrm{V}_{\mathrm{C}}$, given the high antisite energy, the mechanism is assumed to be migration between nearest-neighbor sites on the $\mathrm{C}$ sublattice. This migration barrier is calculated from NEB to be $4.41 \mathrm{eV}$. Similarly, the diffusion of $\mathrm{V}_{\mathrm{Zr}}$ is assumed to occur by nearest-neighbor hops on the $\mathrm{Zr}$ sublattice, and such a hop has a barrier of $5.44 \mathrm{eV}$. As recently identified by Razumovskiy et al.[15], the $\mathrm{V}_{\mathrm{Zr}}-6 \mathrm{~V}_{\mathrm{C}}$ cluster is very stable and has a relatively low migration barrier of $4.54 \mathrm{eV}$. Movement of this cluster is expected to be the dominant mechanism for vacancy-mediated diffusion of $\mathrm{Zr}$. However, this vacancy cluster still has a much lower mobility compared with interstitials, as described below. 
The fast interstitial diffusion is the relevant diffusion mechanism governing the system response to radiation damage and is therefore the relevant mechanism for the analysis of amorphizaton in this paper. We therefore do not consider the vacancy diffusion of clusters further in this work. However, we will investigate the effect of vacancy clusters on the recombination barriers in section 4.2.1.

For the migration of $\mathrm{C}_{\mathrm{i}}$, we found the diffusion path guided by the AIMD simulation. The starting point of the AIMD run is the most stable $\mathrm{C}$ interstitial structure, i.e., a nonlinear C-C-C trimer[2]. We observed that the diffusion path consists of two basic hop steps. The first hop step is a turning hop between the second nearest neighboring $\mathrm{C}_{\mathrm{i}}$ sites with a barrier of $0.27 \mathrm{eV}$. Here the hop is between two C-C-C trimers that share only one common $\mathrm{C}$ atom. Just using this turning hop, the $\mathrm{C}_{\mathrm{i}}$ atom can only jump back and forth within a $\mathrm{C}$ tetrahedron. The long-distance migration needs the second hop step, which is a straight hop between the first nearest neighboring $\mathrm{C}_{\mathrm{i}}$ sites with a barrier of $0.01 \mathrm{eV}$. The hop is between two non-linear C-C-C trimers that share two common $\mathrm{C}$ atoms. The energy landscape and migration path of $\mathrm{C}_{\mathrm{i}}$ is shown in Fig. 1. As shown by the inset of Fig. 1, the $\mathrm{C}_{\mathrm{i}}$ atom (purple ball) migrates downward via a turning hop and diffuses to the right cell through a straight hop. With the combination of these two kinds of hops, the $\mathrm{C}_{\mathrm{i}}$ atom can move arbitrarily long distances and diffuse. The migration barrier of $\mathrm{C}_{\mathrm{i}}$ is mainly determined by the turning hop, which yields a barrier of $0.27 \mathrm{eV}$.

For the diffusion of $\mathrm{Zr}_{\mathrm{i}}$, three diffusion mechanisms have been observed from the AIMD simulations (done at 2800K), (i) Zr interstitialcy mechanism, where the $\mathrm{Zr}$ atom moves to 
the neighboring $\mathrm{Zr}$ site and kicks out the $\mathrm{Zr}$ atom, for which the barrier is $0.47 \mathrm{eV}$; (ii) $\mathrm{Zr}$ interstitial mechanism, where the $\mathrm{Zr}$ atom diffuses from one interstitial site to another interstitial site, for which the barrier is $0.92 \mathrm{eV}$; and (iii) $\mathrm{Zr}$ kicks out C mechanism, where the $\mathrm{Zr}$ atom moves to the neighboring $\mathrm{C}$ site and kicks out a $\mathrm{C}$ atom, for which the barrier is $1.41 \mathrm{eV}$. The most favorable mechanism is the $\mathrm{Zr}$ interstitialcy mechanism, which has the lowest energy barrier of $0.47 \mathrm{eV}$, its energy landscape and migration path is shown in Fig. 2.

The migration barriers of $\mathrm{C}$ and $\mathrm{Zr}$ defects are summarized in Fig. 3. It shows that both the $\mathrm{C}$ and $\mathrm{Zr}$ interstitials have much lower migration barriers than the corresponding vacancies. This indicates that the interstitials are much easier to move than vacancies, as might be expected. Using the migration barrier $E_{m}$ of the most favorable mechanism for each type of point defect, together with the formation energy $E_{f}$ obtained before[2], we calculate the activation energy $E_{a}=E_{m}+E_{f}$ for diffusion of $\mathrm{C}$ and $\mathrm{Zr}$ by interstitial and vacancy mechanisms. These values are listed in Table 1 together with some literature data.

After determining the activation energies $E_{a}$, the diffusion coefficients for each species ( $\mathrm{Zr}$ and $\mathrm{C}$ ) via interstitial and vacancy mediated diffusion were estimated from the following formula,

$$
D=D_{0} \exp \left(Q / k_{B} T\right)
$$

where $Q=E_{a}$ under thermal equilibrium conditions, and $Q=E_{m}$ under irradiated condition as the radiation is assumed to form the defects. The diffusion prefactor is given 
by [25] $D_{0}=g f_{0} a_{0}^{2}{ }_{0} \exp \left[\left(S_{f}+S_{m}\right) / k_{B}\right]$, where $g$ and $f_{0}$ are the geometric factor and correlation factor, respectively (with values $g=1$ and $f_{0}=1$ for the rock-salt structure of $\mathrm{ZrC}), a_{0}$ is the lattice parameter $(\sim 4.72 \AA$ in our DFT calculation $), v_{0}$ is the attempt frequency which we assigned an approximate phonon frequency value of $5^{*} 10^{12} \mathrm{~Hz}[26]$. $\Delta S_{f}$ and $\Delta S_{m}$ are the entropy of defect formation and entropy of migration, respectively. At the thermal condition, $\Delta S_{f}$ and $\Delta S_{m}$ may both contribute but are taken to be 0 to simplify the calculation. At the irradiated condition, only $\Delta S_{m}$ will contribute (since the formation of the defect is assumed to be controlled by radiation produced defects) and is also taken to be 0 . As shown in Table 1 , in our calculation $D_{0}=2.23^{*} 10^{-7} \mathrm{~m}^{2} / \mathrm{s}$, which is similar to that of the intrinsic diffusion of $\mathrm{C}$ in a recent experimental reference[27] but smaller than those in earlier experimental references[16, 28].

The diffusion coefficients of $\mathrm{C}$ and $\mathrm{Zr}$ by interstitial and vacancy mechanisms as functions of temperature are then obtained from Eq. (2) and are shown in Fig. 4 in the temperature range of approximately $500 \mathrm{~K}-2000 \mathrm{~K}$. The experimental results of diffusion coefficients of $\mathrm{C}$ and $\mathrm{Zr}$ from the literature are also plotted as comparison. As shown in Fig. 2, $C$ defects $\left(C_{i}\right.$ and $\left.V_{C}\right)$ have much higher diffusivity than $\mathrm{Zr}$ defects $\left(\mathrm{Zr}_{\mathrm{i}}\right.$ and $\left.\mathrm{V}_{\mathrm{Zr}}\right)$, the diffusion coefficient of $\mathrm{V}_{\mathrm{C}}$ is higher than that of $\mathrm{Zr}_{\mathrm{i}}$ due to the much lower formation energy of $V_{C}[2]$, which leads to a higher concentration of $V_{C}$. For $C$ or $\mathrm{Zr}$ defects, interstitials have higher diffusivity than vacancies. At low temperature, the vacancies are approximately immobile and only interstitials can move. The mobility of interstitials is so high that it is easy to expect interstitials to move around and find vacancies to recombine, 
even at low temperatures. But the actual annealing process is dependent on the recombination barriers, which will be studied in detail in the following sections.

For the stoichiometry conditions $(\mathrm{x} \approx 1)$ calculated here, we predict a much lower activation energy for interstitials than vacancy mediated diffusion, which implies that $\mathrm{C}$ will primarily diffuse by an interstitial mechanism with a barrier of $3.83 \mathrm{eV}$ under thermal conditions. Van Loo et al. [27] found an experimental activation energy of 3.15 $\mathrm{eV}$, reasonably close to what we predicted. However, the experimental activation energy was found to be $4.72 \mathrm{eV}$ and $4.91 \mathrm{eV}$ in $\mathrm{ZrC}_{0.97}[16]$ and $\mathrm{ZrC}_{0.965}$ [28], respectively. Both these values are closer to our predicted migration energy for a vacancy mediated $\mathrm{C}$ transport, which is $5.34 \mathrm{eV}$. It is not clear why these samples, which are quite similar in stoichiometry, should change diffusion mechanisms, but assuming we take $\mathrm{C}$ in the Van Loo et al. work $\left(\mathrm{ZrC}_{x \approx 1}\right)$ [27] to be a pure interstitial diffuser and in $\mathrm{ZrC}_{0.97}[16]$ and $\mathrm{ZrC}_{0.965}[28]$ to be a pure vacancy diffuser, then our predicted activation energies show significant quantitative errors, being too high by $0.68 \mathrm{eV}$ for the interstitials and $0.43-$ $0.62 \mathrm{eV}$ for the vacancies. The vacancy errors are to be expected as they could be due to some remaining contribution from interstitials lowering the experimentally measured activation energy or the $a b$ initio calculation overestimating the $\mathrm{C}$ vacancy formation energy due to the use of C-rich conditions. However, no such obvious mechanisms explain the interstitial errors. The overestimation for all our $\mathrm{C}$ activation barriers could also be in part due to additional vacancies in the experimental system as compared to our stoichiometric computational system weakening the bond strengths, as proposed in Ref. [29], and therefore lowering the migration energies. Another possible source of error is 
the vibrational contribution to the free energy of formation [15]. While the source of the discrepancies could be any of the above, and is certainly to some extent just errors in the DFT approximations, the errors may suggest some additional active mechanisms that have been missed in the modeling, and further study is warranted.

The predicted activation energies for $\mathrm{Zr}$ diffusion mediated by interstitials $(10.83 \mathrm{eV})$ or vacancies $(12.63 \mathrm{eV})$ are much higher than the one reported experimental value of 7.46 $\mathrm{eV}[16]$. As mentioned above, an explanation for the lower experimental value has recently been proposed by Razumovskiy et al.[15] and is based on the $\mathrm{Zr}$ vacancy diffusing as a $\mathrm{V}_{\mathrm{Zr}}-6 \mathrm{~V}_{\mathrm{C}}$ cluster.

\section{Recombination of Frenkel pairs}

\subsection{Energetics of Frenkel pairs in $\mathrm{ZrC}$}

To study the annealing process of point defects, we need to first investigate the energetics of Frenkel pairs in $\mathrm{ZrC}$. In the following, we will focus on the annealing of both $\mathrm{C}$ and $\mathrm{Zr}$ Frenkel pairs in $\mathrm{ZrC}$. The configurations of Frenkel pairs are named based on the relation between the separation $d_{\mathrm{FP}}$ and distances of nearest neighbors. For example, C4thNN represents a $\mathrm{C} F \mathrm{FP}$ with $\mathrm{C}_{\mathrm{i}}$ and $\mathrm{V}_{\mathrm{C}}$ are the fourth nearest neighbors to each other, and $\mathrm{Zr3rdNN}$ represents a $\mathrm{Zr}$ Frenkel pair $\left(\mathrm{Zr}\right.$ FP) with $\mathrm{Zr}_{\mathrm{i}}$ and $\mathrm{V}_{\mathrm{Zr}}$ are the third nearest neighbors to each other. 
The interaction between defects is measured by the binding energy $\left(E_{b}\right)$ of the defect pair, which is defined as the difference between the $E_{f}$ of the defect pair and the sum of the $E_{f}$ of the isolated defects. As an example, we define the binding energy of a C FP as $E_{b}=$ $E_{f}(\mathrm{C} \mathrm{FP})-\left[E_{f}\left(\mathrm{C}_{\mathrm{i}}\right)+E_{f}\left(\mathrm{~V}_{\mathrm{C}}\right)\right]$, where $E_{f}(\mathrm{X})$ is the formation energy of $X$ defect, as defined in the Method section. Note that the negative value of $E_{b}$ means that the Frenkel pair structure is more stable than the single defects. The larger the negative value is, the stronger the attractive interaction is. We define $d_{\mathrm{FP}}$ (in the unit of DFT lattice parameter $\mathrm{a}_{0} \approx 4.72 \AA$ ) to represent the separation between the interstitial and the vacancy of the Frenkel pair, e.g., the distance between $\mathrm{C}_{\mathrm{i}}$ and $\mathrm{V}_{\mathrm{C}}$ in the $\mathrm{C}$ FP. Table 2 lists the formation energies $\left(E_{f}\right)$, binding energies $\left(E_{b}\right)$ and separation $\left(d_{\mathrm{FP}}\right)$ of the related C FPs and $\mathrm{Zr}$ FPs in $\mathrm{ZrC}$. Since $E_{b}$ for $\mathrm{C} 4 \mathrm{thNN}$ is very small $(\sim-0.08 \mathrm{eV})$, the interaction between $\mathrm{C}_{\mathrm{i}}$ and $\mathrm{V}_{\mathrm{C}}$ is negligible, thus we use $\mathrm{C} 4 \mathrm{thNN}$ as a starting point for the C FP recombination. Similarly, $\mathrm{Zr} 7 \mathrm{thNN}$ is chosen as a starting point for the $\mathrm{Zr}$ FP recombination due to its small $E_{b}(\sim 0.07 \mathrm{eV})$.

\subsection{Recombination path and barriers}

\subsubsection{Recombination of C Frenkel pairs}

The first step to study the recombination of Frenkel pairs is to find the recombination path. Since the energy landscape is potentially very complicated, it is hard to get the path intuitively. We therefore used AIMD as a guide to establish the path. From the AIMD simulation, the $\mathrm{C}_{\mathrm{i}}$ of $\mathrm{C} \mathrm{FP}$ was more likely to diffuse away rather than recombine with the 
$\mathrm{V}_{\mathrm{C}}$ directly. We tried several AIMD runs at different temperature with different starting points, such as $\mathrm{C} 3 \mathrm{rdNN}$ ( $\mathrm{NN}=$ nearest neighbor of the vacant site where the recombination occurs) and C4thNN. We observed recombination in the AIMD run with a starting point of C3rdNN at $2800 \mathrm{~K}$. From this AIMD simulation, the $\mathrm{C}_{\mathrm{i}}$ started from the $3 \mathrm{rdNN}$, and diffused to the 4 thNN, and returned to the $3 \mathrm{rdNN}$ again, then passed $2 \mathrm{ndNN}$, and finally recombined with the $\mathrm{V}_{\mathrm{C}}$. From the AIMD movie, $\mathrm{C}_{\mathrm{i}}$ went between $3 \mathrm{rdNN}$ and 4thNN back and forth, which indicates that the hop 3rdNN $\leftrightarrow$ rdNN in both directions is relatively easy but that the barrier to move closer to $V_{C}$ is relatively high. Guided by AIMD, the recombination path was proposed as $4 \rightarrow 3 \rightarrow 2 \rightarrow 0$ (where each number refers to a $\mathrm{C}$ sublattice nearest-neighbor position). The endpoints and intermediate points were fully relaxed as denoted by C4thNN, C3rdNN, C2ndNN, and 0thNN, whose formation energies and binding energies are shown in Table 2. The formation energies of C4thNN and $\mathrm{C} 3 \mathrm{rdNN}$ are similar to each other, and $\mathrm{C} 2 \mathrm{ndNN}$ has a relatively low formation energy. We note that the $\mathrm{C} 1 \mathrm{stNN}$ is not stable, since the $\mathrm{C}_{\mathrm{i}}$ in $\mathrm{C} 1 \mathrm{stNN}$ recombines spontaneously with the $\mathrm{V}_{\mathrm{C}}$.

The barriers of hops between these fully relaxed points were calculated using the NEB method. The whole recombination path $4 \rightarrow 3 \rightarrow 2 \rightarrow 0$ and energy landscape along this path are shown in Fig. 5. The barriers of hops $4 \rightarrow 3(0.38 \mathrm{eV})$ and $3 \rightarrow 2(0.35 \mathrm{eV})$ are comparable to the $\mathrm{C}_{\mathrm{i}}$ migration barrier $\left(E_{m}\left(\mathrm{C}_{\mathrm{i}}\right) \sim 0.27 \mathrm{eV}\right)$. When the $\mathrm{C}_{\mathrm{i}}$ is at the $2 \mathrm{ndNN}$ site, both the barriers to go forward $\left(E_{r} \sim 1.66 \mathrm{eV}\right.$ for hop $\left.2 \rightarrow 0\right)$ and go backward $\left(E_{\uparrow} \sim 1.23\right.$ $\mathrm{eV}$ for hop 2 $\rightarrow 3)$ are much higher than $E_{m}\left(\mathrm{C}_{\mathrm{i}}\right)$. Thus the energy landscape is what has been called a trapping profile $\left(E_{r}>E_{m}, E_{t}>E_{m}\right)[24,30]$ and $\mathrm{C}_{\mathrm{i}}$ is trapped at the $2 \mathrm{ndNN}$ 
site. The recombination barrier is $1.66 \mathrm{eV}$, which is determined by the hop $2 \rightarrow 0$. The recombination barrier is so high that it is not easy to observe recombination of a single $\mathrm{C}$ FP in the AIMD runs.

With so high recombination barrier for a single C FP, models for amorphization based on buildup of point defects destabilizing the crystalline phase would predict that amorphization could occur at lower temperature due to buildup of $\mathrm{C}_{\mathrm{i}}$ and $\mathrm{V}_{\mathrm{C}}$ defects. However, no amorphization has been observed in the literature, which suggests that some mechanisms must occur to lower the barrier for C FP recombination. We note that a critical concentration of defects needs to be accumulated to reach the amorphization criteria[31]. In the presence of multiple defects, the interaction between defects will affect the energy landscape of $\mathrm{C}$ recombination, and we hypothesize that these interactions could reduce the barrier for C FP recombination.

To check the influence of multiple defects interaction, we run AIMD simulations for multiple defects. These configurations with multiple defects were fully relaxed to get their stable structures. For these AIMD runs we used a 64 -atom supercell with a $1 * 1 * 1$ k-point mesh at $1500 \mathrm{~K}$ with duration time of $10 \mathrm{ps}$. First, we studied the system with $8 \mathrm{C}$ FPs, i.e., up to $25 \%$ defects in the system. From AIMD simulation, we observed that $5 \mathrm{C}$ FPs recombined, while the other $3 \mathrm{C}$ FPs did not. This result indicates that the recombination of C FP can be enhanced by the presence of other defects. Our hypothesis is that this enhancement of recombination is mainly from the existence of extra $V_{C}$ rather than $\mathrm{C}_{\mathrm{i}}$. To check the critical role of the $\mathrm{V}_{\mathrm{C}}$, our second test was to run AIMD for $3 \mathrm{C}$ FPs 
with 5 extra $\mathrm{V}_{\mathrm{C}}$, and compared this calculation with the AIMD results of only $3 \mathrm{C}$ FPs without extra $\mathrm{V}_{\mathrm{C}}$. We found that no recombination happens for only $3 \mathrm{C}$ FPs, but 2 of the $3 \mathrm{C}$ FPs recombine in the presence of 5 extra $V_{C}$. This result supports our hypothesis that the existence of extra $V_{C}$ can enhance the recombination of $C$ FPs. To further check this hypothesis, a third AIMD simulation was executed for a single $\mathrm{C}$ FP with 7 extra $\mathrm{V}_{\mathrm{C}}$, then compared with an AIMD run for only a single $\mathrm{C} F \mathrm{FP}$ without extra $\mathrm{V}_{\mathrm{C}}$. No recombination occurred for only a single $C F P$, but the $C_{i}$ recombined with one $V_{C}$ in the presence of 7 extra $\mathrm{V}_{\mathrm{C}}$. Finally, we calculate the recombination barrier of a C FP in the presence of 7 extra $\mathrm{V}_{\mathrm{C}}$ using $\mathrm{NEB}$. The recombination path and energy landscape are shown in Fig. 6. The square represents an extra $V_{C}$. The other extra vacancies are outside of the atomic region shown here. The dark purple balls show the $C_{i}$ positions in the NEB endpoints and images. The presence of extra $V_{C}$ helps $C_{i}$ to find a way to recombine with one $\mathrm{V}_{\mathrm{C}}$ along the path $4 \rightarrow 0$. The barrier along this path is $0.2 \mathrm{eV}$, which is slightly lower than the $\mathrm{C}_{\mathrm{i}}$ migration barrier $(0.27 \mathrm{eV})$ and much lower than the single $\mathrm{C} F \mathrm{FP}$ recombination barrier $(1.66 \mathrm{eV})$. To check whether this path $4 \rightarrow 0$ is only active in the presence of other vacancies, we used NEB to calculate the recombination barrier along this $4 \rightarrow 0$ path for a single $C$ FP without any extra $V_{C}$ in the cell. The barrier was calculated to be $1.88 \mathrm{eV}$, which is higher than the previously determined $1.66 \mathrm{eV}$ barrier. This result indicates that the path found in the presence of other $V_{C}$ for $C F P$ recombination is not a preferable path for the recombination of single C FP. These results demonstrate that the presence of extra $\mathrm{V}_{\mathrm{C}}$ can change the recombination path and reduce the recombination barrier for $\mathrm{C} \mathrm{FP}$ recombination. We refer to this lowering of the recombination barrier due to additional defects as a cluster effect. We note that this 
cluster effect is consistent with the approximately athermal FP recombination seen in Jiang et al.[17] after a large concentration of C FP defects were introduced.

We note that $\mathrm{ZrC}$ is typically quite sub-stoichiometric, with extra vacancies on the $\mathrm{C}$ sublattice $\left(x<1\right.$ in $\left.\mathrm{ZrC}_{x}\right)$ being stable up to $40 \%[6,8]$. In irradiated $\mathrm{ZrC}$, there are expected to be a relatively high concentration of $\mathrm{V}_{\mathrm{C}}$ defects due to there being present initially, the lighter mass of $\mathrm{C}$ vs. $\mathrm{Zr}$ leading to its taking more damage, and the generally much lower formation energy for $\mathrm{V}_{\mathrm{C}}$ than other defects. Therefore, in such samples we expect that the abundant $\mathrm{V}_{\mathrm{C}}$ will enhance $\mathrm{C}$ FP recombination through the cluster effect. We also note that, as discovered recently by Razumovskiy et al.[15], $\mathrm{V}_{\mathrm{Zr}}-\mathrm{nV} \mathrm{V}_{\mathrm{c}}(\mathrm{n}=$ number of $\mathrm{V}_{\mathrm{c}}$ in the cluster) clusters are likely to be a prevalent defect species, and these particular cluster structures might impact recombination. As it is not practical to calculate recombination for all the possible $\mathrm{V}_{\mathrm{Zr}}-\mathrm{nV}$ clusters, we focus here on the $\mathrm{V}_{\mathrm{Zr}}-6 \mathrm{~V}_{\mathrm{C}}$ cluster which was reported to be the most stable by Razumovskiy et al.[15]. From ab initio MD, we observed the recombination path for a $C_{i}$ to recombine with one $V_{C}$ in the $V_{Z r}-6 V_{C}$ cluster. The corresponding barrier was calculated to be about $0.14 \mathrm{eV}$, which is lower than the migration barrier of $\mathrm{C}_{\mathrm{i}}(0.27 \mathrm{eV})$. Thus for the $\mathrm{V}_{\mathrm{Zr}}-6 \mathrm{~V}_{\mathrm{C}}$ cluster we again see a cluster effect reducing the recombination barrier. The reduced barriers for C FP recombination in the presence of vacancy clusters, either from irradiation, defect clustering, or due to intrinsic off-stoichiometry, suggests that C FPs will be able to recombine easily before they can build up and amorphize the material. These calculated C FP recombination energetics and the cluster effect help to explain the observation that $\mathrm{ZrC}$ has a high RTA. 


\subsubsection{Recombination of Zr Frenkel pairs}

We used a similar method that described for C FPs to study the Zr FP recombination. After full relaxation, we obtained the stable configurations for $\mathrm{Zr} 7 \mathrm{thNN}$, Zr6thNN, and Zr3rdNN. For Zr5thNN, Zr4thNN, Zr2ndNN and Zr1stNN, the Zr interstitial moved to one of the other neighbor sites or recombined. Then AIMD simulations were performed for the three stable $\mathrm{Zr}$ FPs, and the recombination paths were observed for $\mathrm{Zr} 7 \mathrm{thNN}$ and $\mathrm{Zr3rdNN}$ starting points (no recombination was observed for Zr6thNN so we assume the barriers for recombination from this site are larger than the other paths and that this path does not play a significant role in the recombination). The NEB calculations were conducted along each of the two observed recombination paths. Here we consider in detail the recombination in the $\mathrm{Zr} 7 \mathrm{thNN}$ as it has lower $E_{b}$ than $\mathrm{Zr} 3 \mathrm{rdNN}$ and is therefore perhaps a more likely recombination path. From the AIMD simulation, one $\mathrm{Zr}_{\mathrm{i}}$ atom ( $\mathrm{Zr} 91$, the number is from the atom index in simulation supercell) of the $\mathrm{Zr}-\mathrm{Zr}$ dumbbell (Zr91-Zr104) started from the 7thNN site, then kicked out another $\mathrm{Zr}$ atom (Zr68) at the 2ndNN site and replaced it. Finally $\mathrm{Zr68}$ recombined with the $\mathrm{V}_{\mathrm{Zr}}$. Thus the recombination path of $\mathrm{Zr} \mathrm{FP}$ is through an interstitialcy mechanism $\left(\mathrm{Zr}_{\mathrm{i}}\right.$ kicks out another $\mathrm{Zr}$ atom) and is denoted as $7 \rightarrow 2 / 2^{\prime} \rightarrow 0$.

The barrier along this $7 \rightarrow 2 / 2^{\prime} \rightarrow 0$ path was calculated through the NEB method. The two endpoints of NEB calculation were set up as follows, $\mathrm{Zr}_{\mathrm{i}}(\mathrm{Zr} 91)$ and $\mathrm{V}_{\mathrm{Zr}}$ were the $7 \mathrm{thNN}$ to each other in the initial state, and the Zr68 atom (kicked out by $\mathrm{Zr91}$ ) recombined with 
the $\mathrm{V}_{\mathrm{Zr}}$ in the final state. The energies of these NEB endpoints are given in Table 2. The energy landscape for the $\mathrm{Zr}$ FP recombination along the $7 \rightarrow 2 / 2^{\prime} \rightarrow 0$ path is shown in Fig. 7. The recombination barrier is about $0.32 \mathrm{eV}$, which is slightly smaller than the migration barrier of $\mathrm{Zr}_{\mathrm{i}}\left(E_{m}\left(\mathrm{Zr}_{\mathrm{i}}\right) \sim 0.47 \mathrm{eV}\right)$, thus the $\mathrm{Zr}$ FP recombination is rate-limited by the migration barrier $E_{m}\left(\mathrm{Zr}_{\mathrm{i}}\right)$. Similarly, for the case of $\mathrm{Zr} 3 \mathrm{rdNN}$, the recombination path is along $3 \rightarrow 2 / 2^{\prime} \rightarrow 0$ based on the AIMD simulation and the recombination barrier from the NEB calculation is about $0.61 \mathrm{eV}$. It is not very difficult for $\mathrm{Zr}$ FP to recombine, and we do not check in more detail for cluster effects analogous to those seen for C FP recombination. We also note that the results of Razumovskiy et al.[15] suggest that the $\mathrm{V}_{\mathrm{Zr}}$ can be decorated by up to $6 \mathrm{~V}_{\mathrm{C}}$. Given the $\mathrm{Zr}_{\mathrm{i}}$ moves without use of the $\mathrm{C}$ sublattice there is no strong reason to believe that this recombination will be impacted significantly by the presence of $\mathrm{C}$ vacancies, although the recombination barrier will probably change somewhat. We therefore assume that the recombination of $\mathrm{Zr}$ FPs has a low barrier independent of how many $\mathrm{C}$ vacancies decorate the $\mathrm{Zr}$ vacancy. Therefore, the $\mathrm{Zr}$ FPs can be easily annealed out due to their low recombination barrier. Considering the cluster effects, C FP can be partially annihilated. Putting these together, we predict that it is hard to reach the critical defect concentration for amorphization in $\mathrm{ZrC}$.

\section{Resistance to amorphization of $\mathrm{ZrC}$}

In the section above we explored the $\mathrm{RTA}$ of $\mathrm{ZrC}$ from the perspective of defect kinetics. In the following we will try to understand $\mathrm{RTA}$ of $\mathrm{ZrC}$ in a broader view based on various amorphization models. In general, amorphization is a transformation in which 
existing long-range orientational order and translational periodicity are lost[32]. The amorphization of materials has been investigated from many perspectives, including those related to structural, chemical, thermodynamic, and kinetic properties. Not surprisingly, a variety of models for amorphization have been proposed, as listed in Table 3. There are several criteria for amorphization, such as free energies[31], volume expansion[33], chemical disorder[34] and stress[35]. Below we consider $\mathrm{ZrC}$ in light of each model and discuss whether its RTA is consistent with that model. This exercise is not aimed at supporting one model or another, but just showing how the RTA of ZrC can be understood from all these different perspectives.

\section{(1) Topology models}

L. W. Hobbs et al. proposed a topology model by setting up a correlation between the susceptibility to irradiation-induced amorphization and the calculated structural freedom $f$, as described in Refs $[32,36-38]$. The lower the value of $f$ is, the harder the amorphization is. This model works well for a wide range of ceramic materials, although $\mathrm{SiC}$ is somewhat poorly modeled by this approach.

$\mathrm{ZrC}$ has a rock-salt structure, which gives a structural freedom $f=-10$. It indicates that this structure is overconstrained and unlikely to form topological disorder[32]. In fact, the rock-salt structure was regarded as the most difficult structure to amorphize based on the topology model[32]. Therefore, Hobbs' topological model would predict that $\mathrm{ZrC}$ is extremely hard to amorphize. 
(2) Thermodynamic models

From the thermodynamic point of view, amorphization was regarded as qualitatively related to the ease of glass formation[39]. S. X. Wang proposed a glass-forming ability model and developed the susceptibility to amorphization, denoted as $S$, which is based on geometric constraints, bonding, and phase transition temperature[40]. While application of their model does suggest $\mathrm{SiC}$ would be easier to amorphize than $\mathrm{ZrC}$, their model was developed for ionic systems and depends strongly on field strengths from ions. It is therefore not clear that their model can be applied to covalent systems like $\mathrm{ZrC}$ and $\mathrm{SiC}$.

Melting temperature $\left(T_{\mathrm{m}}\right)[42]$ and the temperature ratio of recrystallization temperature ( $T_{\text {crystal }}$ ) to $T_{\mathrm{m}}$ [43] have also been considered as parameters for assessing RTA. Although a recrystallization temperature of $\mathrm{ZrC}$ has not been measured as far as we are aware, $T_{m}$ of $\mathrm{ZrC}$ is very high, about $3700 \mathrm{~K}[2]$, which is consistent with a high RTA. But the limitations of simple models depending on these parameters are (i) high $T_{\mathrm{m}}$ does not always corresponds to high RTA (e.g., $\mathrm{SiC}$ is a counter example), and (ii) $T_{\text {crystal }}$ is not a fundamental parameter, since it has a wide range depending the specific measurement conditions rather than an intrinsic value.

In a related type of thermodynamic analysis, researchers have used enthalpies of the defected crystal and amorphous phase relative to the perfect crystal to measure RTA[33, 44-45]. However, these methods are limited as they ignore any type of kinetic 
contributions and require significant knowledge of the amorphous phase thermodynamics (e.g., enthalpy and Deby temperature), so we do not consider them further here.

\section{(3) Chemical models}

From the perspective of chemical properties, $\mathrm{K}$. Trachenko proposed that the resistance to amorphization of a non-metallic material is determined by the competition between the short-range covalent and long-range ionic forces[46]. Higher ionicity or less covalency of a material makes it harder to amorphize, as the ability to form a covalent network leads to damage stabilization and makes a material amorphizable. In $\mathrm{ZrC}$, the strong covalent metal-nonmetal bonding dominates, although there is also some ionic bonding and nonnegligible metallic bonding[7]. However, the primarily covalent character of $\mathrm{ZrC}$ means that the Trachenko model would predict relatively low RTA, unlike what is absorbed. However, ZrC is metallic which may be why Trachenko's model, which is for nonmetallic systems, does not give good qualitative guidance.

The idea that chemical disorder is a driving force for amorphization was proposed, e.g., in Ref. [34], and might be used to explain the high RTA of $\mathrm{ZrC}$. In $\mathrm{ZrC}$, the formation energies of antisites are very high[2] and we expect few to form thermally or under irradiation, consistent with results of simulations of collision cascades in $\mathrm{ZrC}[47]$. Therefore, it is unlikely that $\mathrm{ZrC}$ can amass significant chemical disorder under irradiation, which is consistent with its high RTA. 
(4) Kinetic models

From the kinetic perspective, amorphization can occur homogeneously or heterogeneously[48]. Homogeneous amorphization can be described by pure defect accumulation model[49]. Heterogeneous amorphization are associated with direct-impact (in cascade)[50-51], local accumulation of defects (cascade-overlap)[50], nucleation and growth processes[52], etc. Many recent models are a combination of these models, and take into account some thermal recrystallization/recovery processes[48, 53-54] or defect stimulated effects[55]. The rate of amorphization depends on damage production and recovery processes under any given irradiation conditions. If damage is produced slowly, or crystallization/nucleation/annealing rates are high, the material is hard to amorphize[48]. If the mobility of defects is fast[31], which leads to high recombination probability, the RTA will also be high. Thus two important ab initio parameters in the kinetic models for amorphization are migration barriers and recombination barriers, with higher RTA consistent with lower barriers[48].

The interstitials in $\mathrm{ZrC}$ have high mobilities due to their low migration barriers $\left(E_{m}\left(\mathrm{C}_{\mathrm{i}}\right) \approx\right.$ $\left.0.27 \mathrm{eV}, E_{m}\left(\mathrm{Zr}_{\mathrm{i}}\right) \approx 0.47 \mathrm{eV}\right)$. While the C FP recombination barrier is significant for isolated FPs, it becomes very low due to the cluster effect, as discussed. Regardless of the cluster effect (as discussed above), the $\mathrm{Zr}$ recombination barrier is also very low. These low recombination barriers make recovery of defects straightforward in $\mathrm{ZrC}$, and therefore a kinetic based model of defect accumulation is consistent with its high RTA. 
Finally, we note that C. Jiang et al.[17] hypothesized that the amorphization process in an compound material could be related to the mechanical stability of the sublattice with the least damage. This picture suggests that for systems where one sublattice is being highly damaged, a stable second sublattice can keep the system from amorphizing. This model was developed to explain differences of $\mathrm{SiC}$ and $\mathrm{ZrC}$ and is therefore quite consistent with the differences between these materials. In this model stable $\mathrm{Zr}$ fcc sublattice is what provides the strong RTA for $\mathrm{ZrC}$.

\section{Conclusion}

We used ab initio methods to investigate the migration of point defects, recombination of Frenkel pairs, and resistance to amorphization in $\mathrm{ZrC}$. We explored diffusion mechanisms for $\mathrm{C}$ and $\mathrm{Zr}$ point defects and calculated the diffusion coefficients based on the migration barriers of intrinsic point defects. Interstitials have lower migration barriers than vacancies, and $\mathrm{C}$ defects have higher diffusivity than $\mathrm{Zr}$ defects. Even though the recombination barrier of a single C FP is high, the recombination barrier is reduced by the presence of extra $\mathrm{V}_{\mathrm{C}}$. The $\mathrm{Zr}$ FP recombination has a low barrier and is rate-limited by the $\mathrm{Zr}_{\mathrm{i}}$ migration barrier. These predictions are consistent with easy defect recombination and high RTA in $\mathrm{ZrC}$. We also considered other amorphization theories and point out that the RTA to amorphization of $\mathrm{Zr}$ could be due to its rock-salt structure, high melting temperature, low susceptibility to glass formation, resistance to chemical disorder, and stability of the $\mathrm{Zr}$ sublattice. 


\section{Acknowledgments}

This research was supported by the DOE Office of Nuclear Energy's Nuclear Energy University Programs (NEUP 10-679). This work benefitted from the use of the Extreme Science and Engineering Discovery Environment (XSEDE), which is supported by National Science Foundation grant number OCI-1053575. The authors acknowledge Prof. Arthur Motta, Prof. Todd Allen, Christopher J. Ulmer, and Dr. Yina Huang for useful discussions. 


\section{Tables}

Table 1. Formation energies $\left(E_{f}\right)$, migration barriers $\left(E_{m}\right)$ and activation energies $\left(E_{a}\right)$ of diffusion via different intrinsic point defects in $\mathrm{ZrC}$. The values of prefactor $D_{0}$ are also listed[6]. Entropies of formation $\left(\Delta \mathbf{S}_{\mathbf{f}}\right)$ and migration $\left(\Delta \mathbf{S}_{\mathbf{m}}\right)$ are set to zero.

\begin{tabular}{|c|c|c|c|c|c|c|c|}
\hline $\begin{array}{l}\text { Mediating } \\
\text { Defect }\end{array}$ & $E_{f}(\mathrm{eV})$ & $E_{m}(\mathrm{eV})$ & $E_{a}(\mathrm{eV})$ & $\begin{array}{c}E_{a} \text { in Expt. } \\
(\mathrm{eV})\end{array}$ & $\begin{array}{c}\Delta \mathbf{S}_{\mathbf{f}} \\
(\mathrm{eV} / \mathrm{K})\end{array}$ & $\begin{array}{c}\Delta \mathbf{S}_{\mathrm{m}} \\
(\mathrm{eV} / \mathrm{K}) \\
\end{array}$ & $\begin{array}{c}\mathbf{D}_{0} \\
\left(\mathbf{m}^{2} / \mathbf{s}\right) \\
\end{array}$ \\
\hline \multicolumn{8}{|l|}{ C Diffusion } \\
\hline $\mathrm{C}$ interstitial & $\begin{array}{c}3.56 \\
\text { (C-rich) }\end{array}$ & 0.27 & 3.83 & & 0 & 0 & 2.23E-7 \\
\hline $\mathrm{C}$ vacancy & $\begin{array}{c}0.93 \\
\text { (C-rich) }\end{array}$ & 4.41 & 5.34 & $\begin{array}{c}4.91[28] \\
\left(\mathrm{ZrC}_{0.965}\right) \\
4.72[16] \\
\left(\mathrm{ZrC}_{0.97}\right) \\
3.15[27] \\
\left(\mathrm{ZrC}_{1}\right. \\
\text { extrapolated })\end{array}$ & 0 & 0 & $\begin{array}{c}1.32 \mathrm{E}-2 \\
{[28]} \\
1.41 \mathrm{E}-3 \\
{[16]} \\
8.3 \mathrm{E}-7 \\
{[27]}\end{array}$ \\
\hline \multicolumn{8}{|l|}{$\mathbf{Z r}$} \\
\hline \multicolumn{8}{|l|}{ Diffusion } \\
\hline $\begin{array}{l}\mathrm{Zr} \\
\text { interstitial }\end{array}$ & $\begin{array}{c}10.36 \\
\text { (C-rich) }\end{array}$ & 0.47 & 10.83 & & 0 & 0 & \\
\hline $\mathrm{Zr}$ vacancy & $\begin{array}{c}7.19 \\
\text { (C-rich) }\end{array}$ & 5.44 & 12.63 & $\begin{array}{l}7.46[16] \\
\left(\mathrm{ZrC}_{0.97}\right)\end{array}$ & 0 & 0 & $\begin{array}{c}1.03 \mathrm{E}-1 \\
{[16]}\end{array}$ \\
\hline $\begin{array}{l}\mathrm{V}_{\mathrm{Zr}}-6 \mathrm{~V}_{\mathrm{C}} \\
\text { cluster[15] }\end{array}$ & $\begin{array}{c}2.42[15] \\
\text { (*Not } \\
\text { C-rich) }\end{array}$ & $4.54[15]$ & $7.46[15]$ & & & & \\
\hline
\end{tabular}

* This formation energy is effectively calculated assuming carbon vacancies have zero formation energy. Such an assumption can make sense when $\mathrm{C}$ vacancies are readily available in $\mathrm{ZrC}_{x}(x<1)$ but this condition does not correspond to the C-rich reference state used in our calculations. 
Table 2. Formation energies $\left(E_{f}\right)$, binding energies $\left(E_{b}\right)$ and separation $\left(d_{\mathrm{FP}}\right)$ of various $\mathrm{C}$ Frenkel pairs in $\mathrm{ZrC}$.

\begin{tabular}{lccc}
\hline Configuration & $\boldsymbol{E}_{f}(\mathbf{e V})$ & $\boldsymbol{E}_{\boldsymbol{b}}(\mathbf{e V})$ & $\boldsymbol{d}_{\mathrm{FP}}\left(\boldsymbol{a}_{\boldsymbol{0}}\right)$ \\
\hline 0thNN & 0.00 & -4.35 & 0.00 \\
C FP (C-rich) & & & \\
C2ndNN & 3.37 & -0.98 & 0.49 \\
C3rdNN & 4.25 & -0.10 & 0.90 \\
C4thNN & 4.27 & -0.08 & 0.99 \\
Zr FP (C-rich) & & & \\
Zr3rdNN & 16.41 & -0.59 & 0.85 \\
Zr7thNN & 17.07 & 0.07 & 1.19 \\
\hline
\end{tabular}


Table 3. Summary of amorphization models, related parameters and limitations.

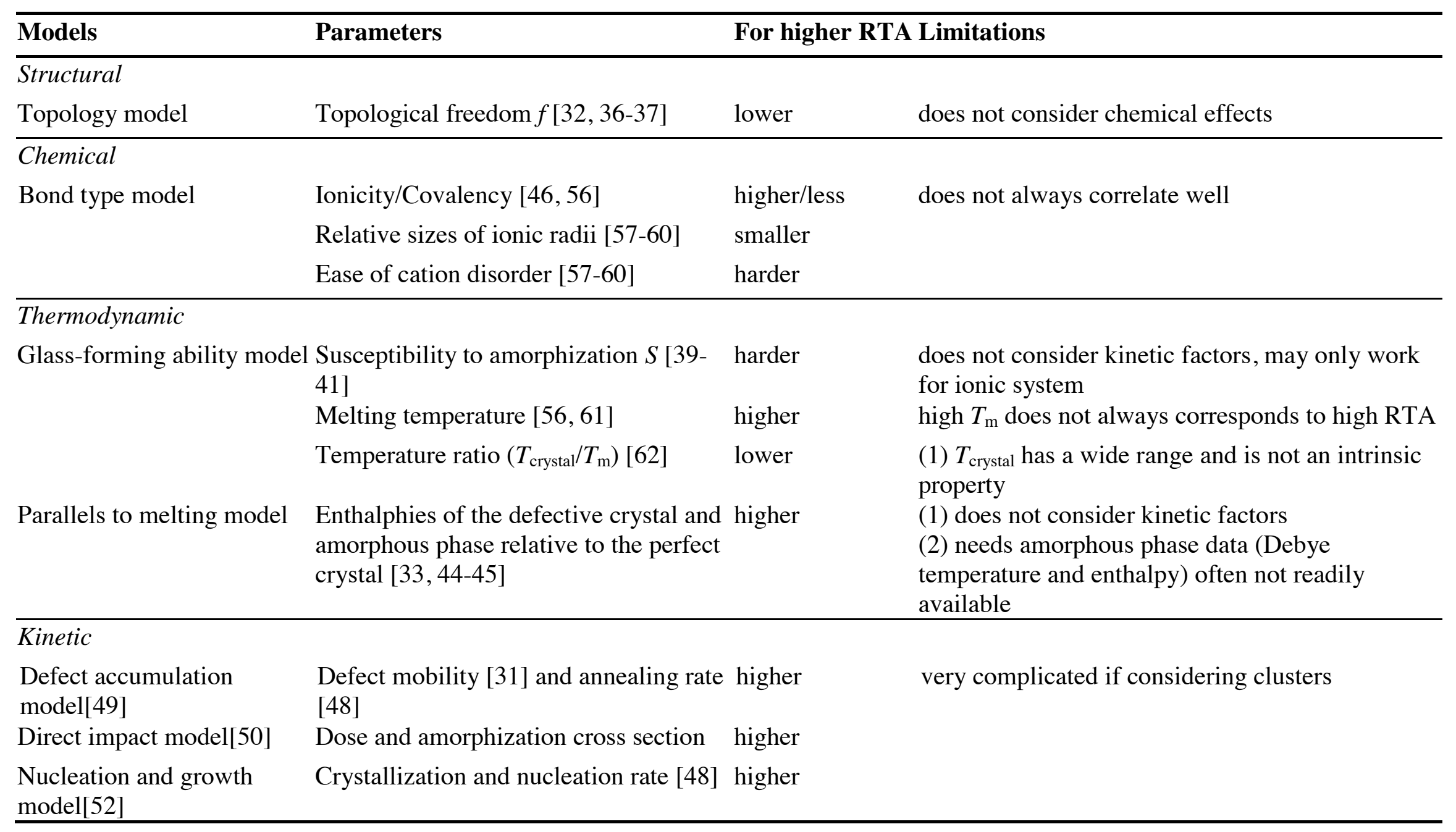




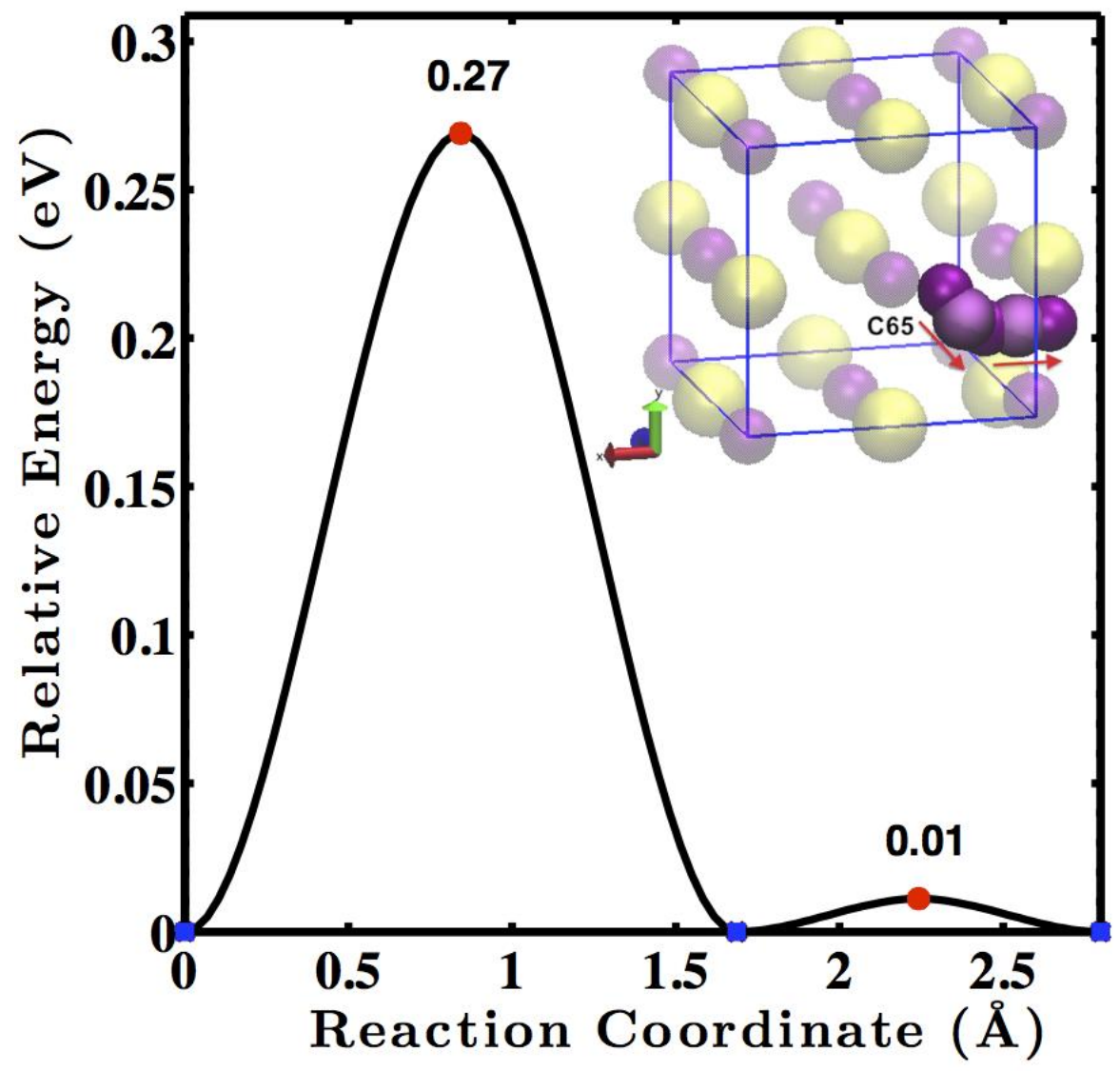

Figure 1. The energy landscape and migration path of $\mathrm{C}_{\mathrm{i}}$ in $\mathrm{ZrC}$. The dark purple and pink small balls represent $\mathrm{C}$ interstitial atom $\mathrm{C} 65$ (65 is the atom index number in the simulation supercell) in the NEB endpoints and images, respectively. The other light purple small balls and light yellow big balls represent the rest of the $\mathrm{C}$ and $\mathrm{Zr}$ atoms, respectively. In the energy landscape, the blue squares and red dots represent the endpoints and barriers, respectively. The marked barriers are with respect to the energy of the closest starting points along the reaction coordinate. 


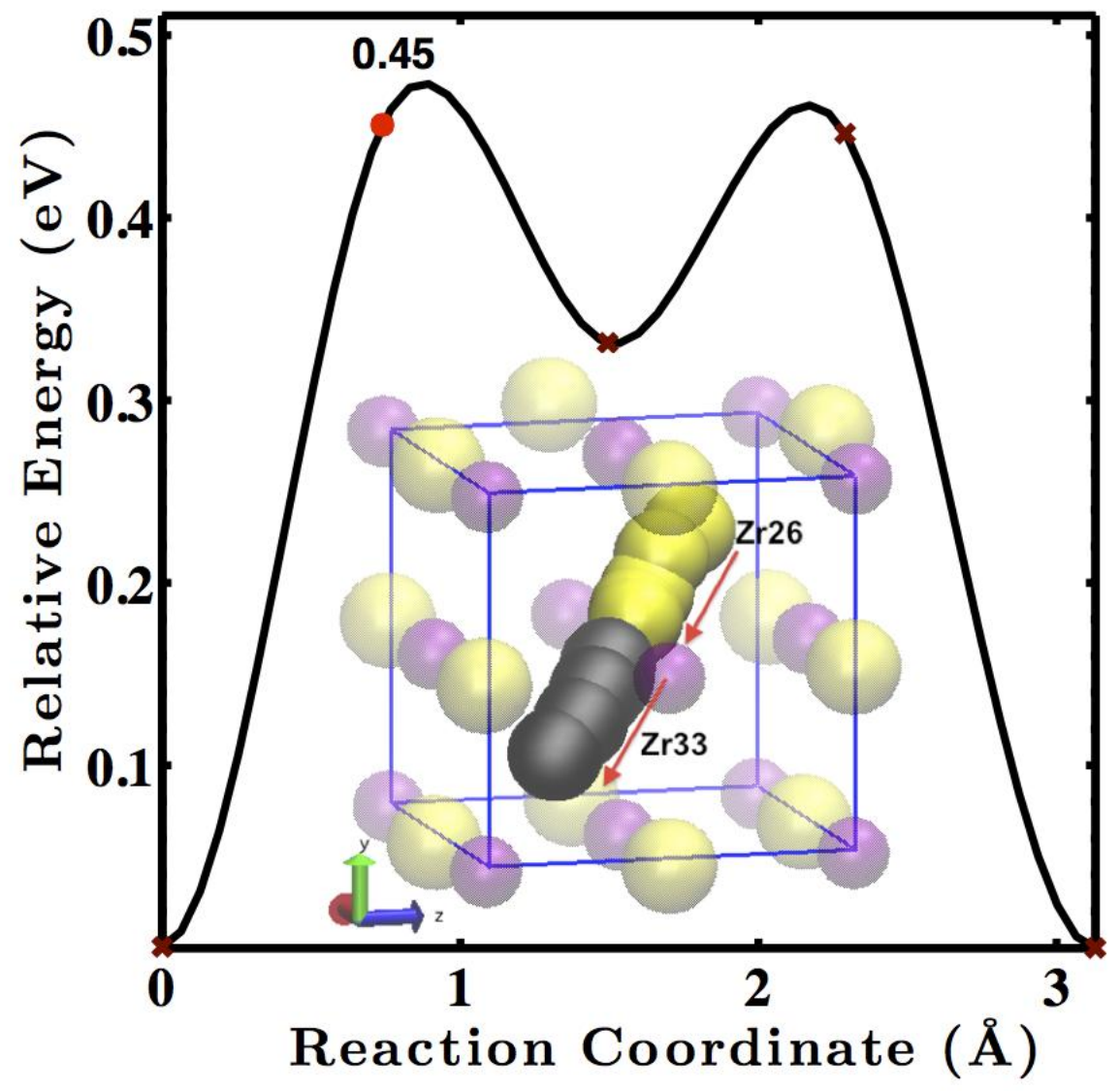

Figure 2. The energy landscape and migration path of $\mathrm{Zr}_{i}$ following the $\mathrm{Zr}$ interstitialcy mechanism in $\mathrm{ZrC}$. The bright yellow and black big balls are the $\mathrm{Zr}$ interstitial atom $\mathrm{Zr} 26$ and the kicked-out $\mathrm{Zr}$ atom $\mathrm{Zr} 33$ in the NEB runs, respectively. The other light yellow big balls and light purple small balls represent the rest of the $\mathrm{Zr}$ and $\mathrm{C}$ atoms, respectively. In the energy landscape, the red dot and brown crosses represent the barriers and images, respectively. 


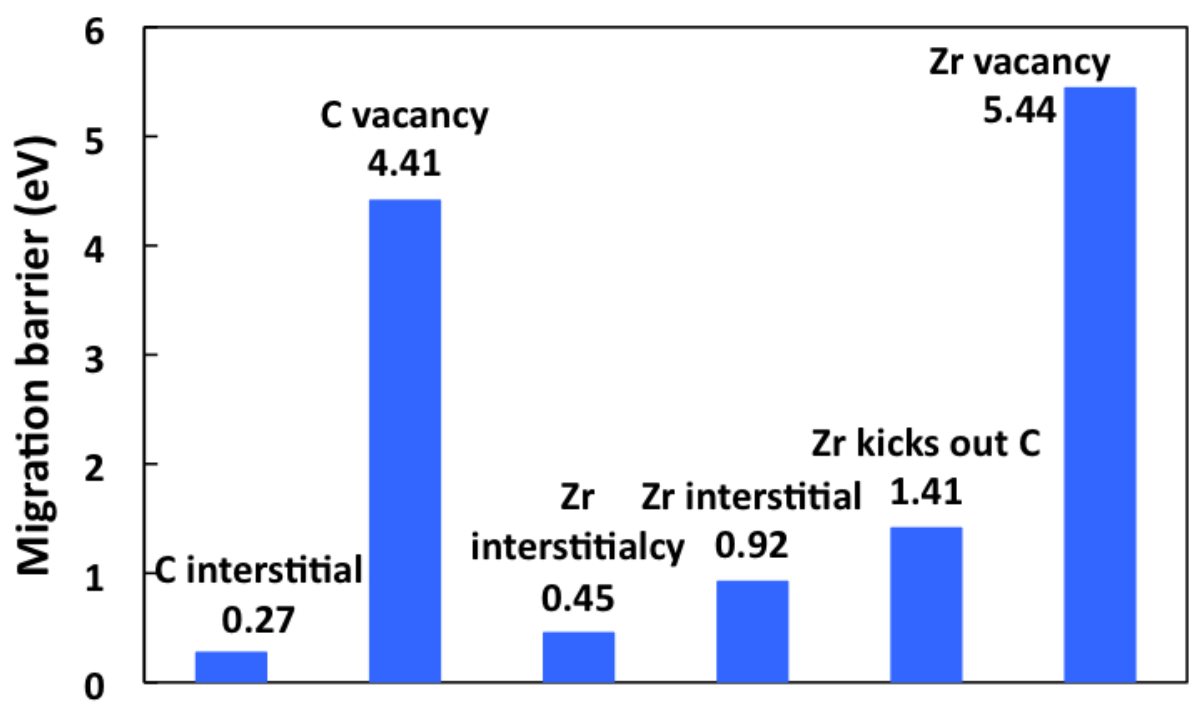

Figure 3. Migration barriers of point defects following various diffusion mechanisms in $\mathrm{ZrC}$. 


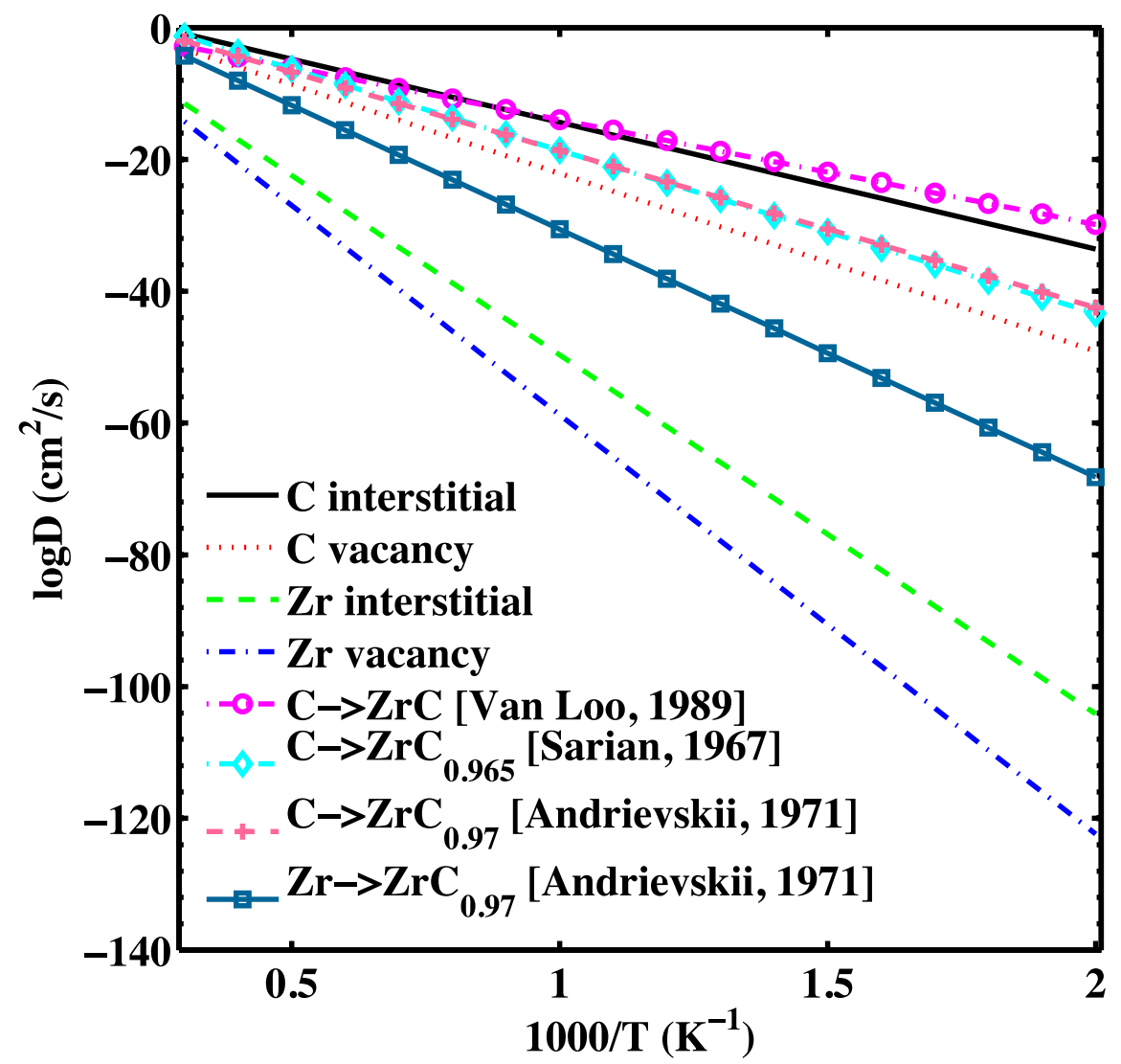

Figure 4. Diffusion coefficients of $\mathrm{C}$ interstitial (solid line), $\mathrm{C}$ vacancy (dotted line), $\mathrm{Zr}$ interstitial (dashed line), and $\mathrm{Zr}$ vacancy (dash dotted line) in $\mathrm{ZrC}$ from the present work, compared with the diffusion coefficients measured in literature. $\mathrm{C}->\mathrm{ZrC}_{x}$ means that the diffusion coefficient of $\mathrm{C}$ in $\mathrm{ZrC}_{x}$. 


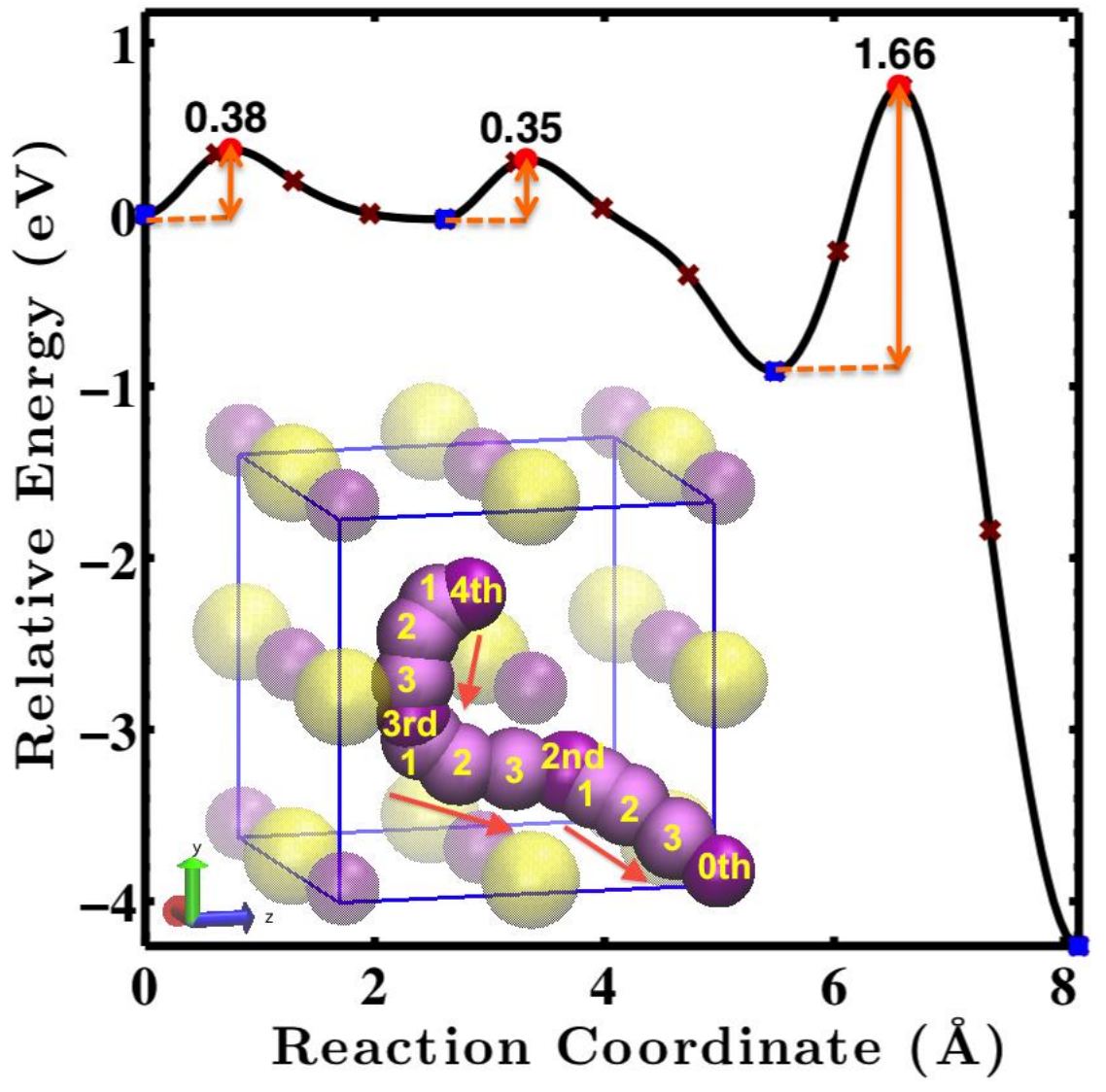

Figure 5. Energy landscape of $\mathrm{C} F \mathrm{FP}$ recombination in $\mathrm{ZrC}$. The recombination path $4 \rightarrow 3 \rightarrow 2 \rightarrow 0$ is shown in the inset. The dark purple and pink small balls represent $C_{i}$ atoms in the NEB endpoints and images, respectively. The other light purple small balls and light yellow big balls represent the rest of the $\mathrm{C}$ and $\mathrm{Zr}$ atoms, respectively. In the energy landscape, the blue squares, red dots, and brown crosses represent the endpoints, barriers, and images, respectively. The marked barriers are with respect to the energy of the closest starting points along the reaction coordinate. 


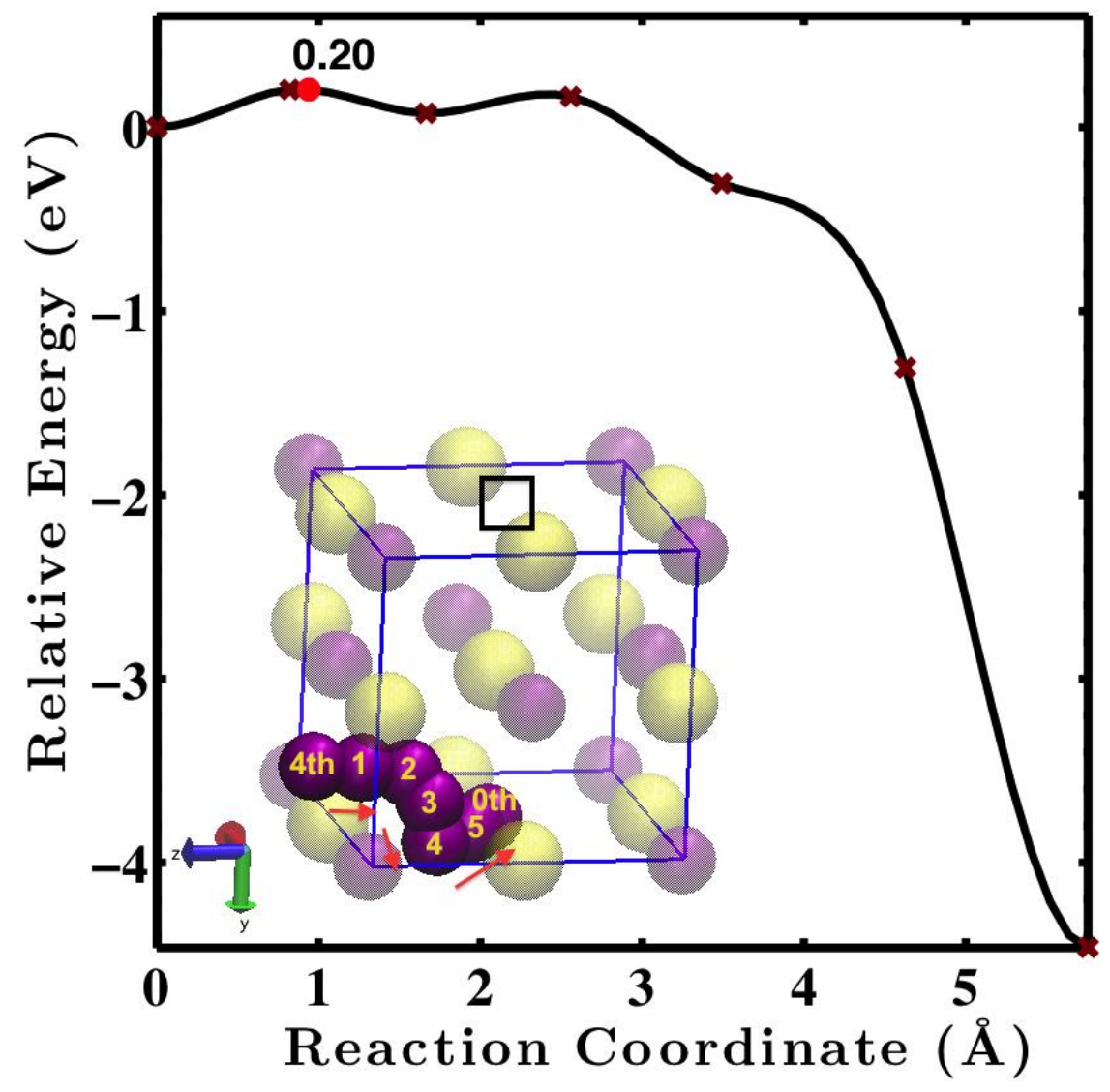

Figure 6. Energy landscape of C FP recombination in the presence of 7 extra $V_{C}$. The recombination path $4 \rightarrow 0$ is shown in the inset. The dark purple small balls are the $C_{i}$ atoms in the endpoints and images of the NEB run. The square represents one of the 7 extra $\mathrm{V}_{\mathrm{C}}$. The other light purple small balls and light yellow big balls represent the rest of the $\mathrm{C}$ and $\mathrm{Zr}$ atoms, respectively. In the energy landscape, the red dot and brown crosses represent the maximum energy point and images, respectively. The marked barriers are with respect to the energy of the closest starting points along the reaction coordinate. 


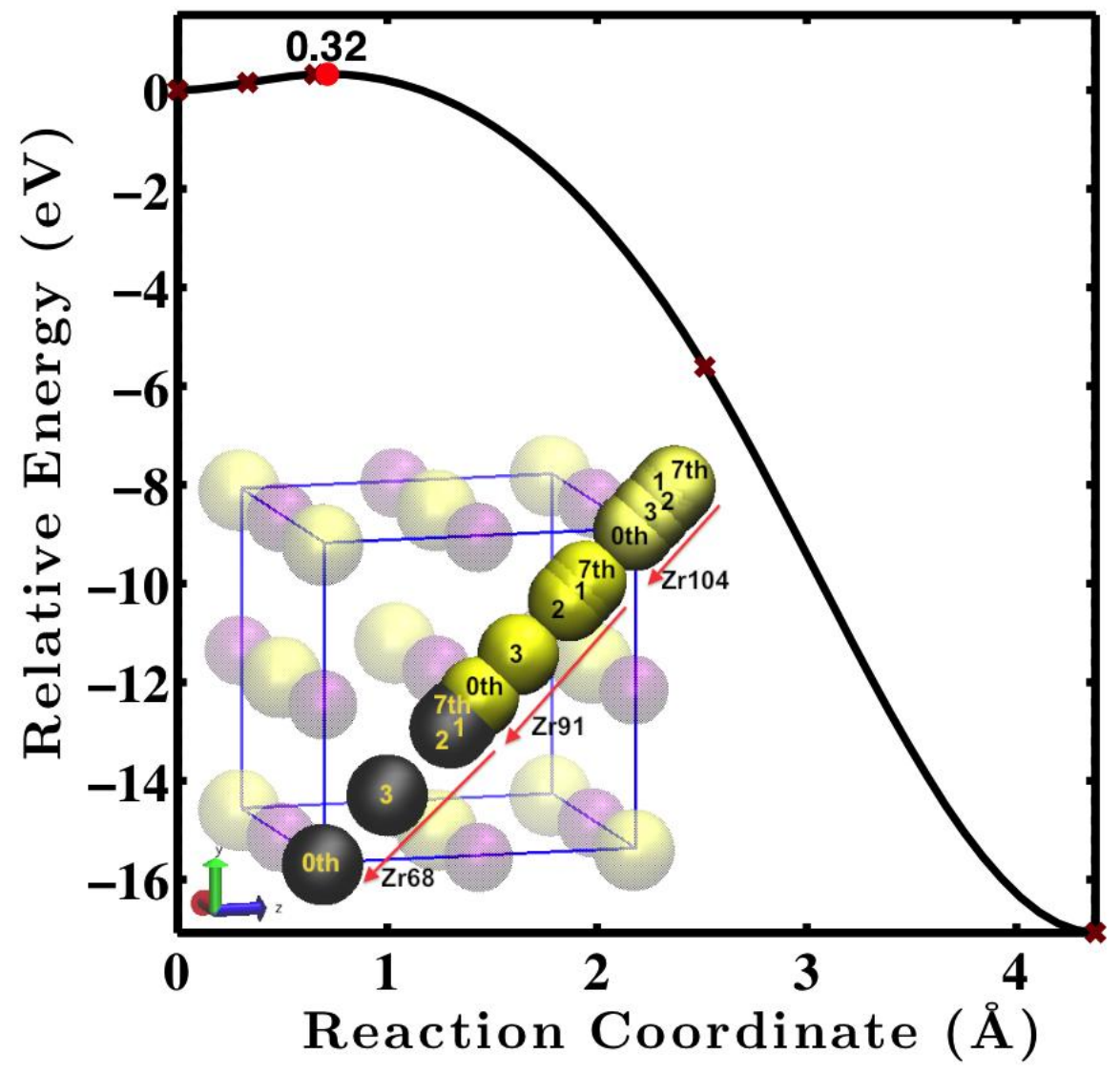

Figure 7. Energy landscape of $\mathrm{Zr}$ FP recombination in $\mathrm{ZrC}$. The recombination path $7 \rightarrow 2 / 2^{\prime} \rightarrow 0$ is shown in the inset. The bright yellow and black big balls are the $\mathrm{Zr}$ dumbbell interstitial atoms $\mathrm{Zr} 91-\mathrm{Zr} 104$ and the kicked-out $\mathrm{Zr}$ atom $\mathrm{Zr68}$ in the NEB runs, respectively. The other light yellow big balls and light purple small balls represent the rest of the $\mathrm{Zr}$ and $\mathrm{C}$ atoms, respectively. In the energy landscape, the red dot and brown crosses represent the barriers and images, respectively. 


\section{References}

1. $\quad$ Sara, R. V., J. Am. Ceram. Soc., 48 (1965) 243-247.

2. $\quad$ Kim, S.; Szlufarska, I.; Morgan, D., J. Appl. Phys., 107 (2010) 053521.

3. Snead, L. L.; Katoh, Y.; Kondo, S., J. Nucl. Mater., 399 (2010) 200-207.

4. Reynolds, G. H.; Janvier, J. C.; Kaae, J. L.; Morlevat, J. P., J. Nucl. Mater., 62 (1976) 9-16.

5. $\quad$ Porter, I. E.; Knight, T. W.; Dulude, M. C.; Roberts, E.; Hobbs, J., Nucl. Eng. Des., 259 (2013) 180-186.

6. Jackson, H. F.; Lee, W. E.; Editor-in-Chief: Rudy, J. M. K., 2.13 - Properties and Characteristics of ZrC. In Comprehensive Nuclear Materials, Elsevier: Oxford, 2012; pp 339-372.

7. Weber, W., Phys. Rev. B, 8 (1973) 5082-5092.

8. $\quad$ Fernandez Guillermet, A., J. Alloys Compd., 217 (1995) 69-89.

9. Gosset, D.; Dolle, M.; Simeone, D.; Baldinozzi, G.; Thome, L., J. Nucl. Mater., 373 (2008) 123-129.

10. Snead, L. L.; Bell, G. L.; Besmann, T. M.; Collins, E. D., D. A. report, (2010).

11. Gosset, D.; Dolle, M.; Simeone, D.; Baldinozzi, G.; Thome, L., Nucl. Instrum. Methods Phys. Res., Sect. B, 266 (2008) 2801-2805.

12. Yang, Y.; Dickerson, C. A.; Swoboda, H.; Miller, B.; Allen, T. R., J. Nucl. Mater., 378 (2008) 341-348.

13. Gan, J.; Meyer, M. K.; Birtcher, R. C.; Allen, T. R., ASTM Spec. Tech. Publ., (2005).

14. Li, J.; Lia, D.; Yip, S.; Najafabad, R.; Ecker, L., J. Appl. Phys., 93 (2003) 9072.

15. Razumovskiy, V. I.; Ruban, A. V.; Odqvist, J.; Korzhavyi, P. A., Phys. Rev. B, 87 (2013) 054203.

16. Andrievskii, R. A.; Khromov, Y. F.; Alekseeva, I. S., Fiz. Met. Metalloved., 32 (1971) 664-7.

17. Jiang, C.; Zheng, M.-J.; Morgan, D.; Szlufarska, I., Phys. Rev. Lett., 111 (2013) 155501.

18. Kresse, G.; Furthmuller, J., Computational Materials Science, 6 (1996) 15-50.

19. Kresse, G.; Furthmuller, J., Phys. Rev. B, 54 (1996) 11169-11186.

20. Kresse, G.; Hafner, J., Phys. Rev. B, 47 (1993) 558-561.

21. Kresse, G.; Hafner, J., Phys. Rev. B, 49 (1994) 14251-14269.

22. $\quad$ Perdew, J. P.; Burke, K.; Ernzerhof, M., Phys. Rev. Lett., 77 (1996) 3865-3868.

23. Henkelman, G.; Uberuaga, B. P.; Jonsson, H., J. Chem. Phys., 113 (2000) 99019904.

24. Zheng, M.-J.; Swaminathan, N.; Morgan, D.; Szlufarska, I., Phys. Rev. B, 88 (2013) 054105.

25. Van der Ven, A.; Ceder, G.; Asta, M.; Tepesch, P. D., Phys. Rev. B, 64 (2001) 184307.

26. Vineyard, G. H., J. Phys. Chem. Solids, 3 (1957) 121-127.

27. Van Loo, F. J. J.; Wakelkamp, W.; Bastin, G. F.; Metselaar, R., Solid State Ionics, 32-33, Part 2 (1989) 824-832.

28. Sarian, S.; Criscione, J. M., J. Appl. Phys., 38 (1967) 1794. 
29. Upadhyaya, G. S., Nature and Properties of Refractory Carbides. Nova Science Publishers: Commack, N.Y., 1986.

30. Swaminathan, N.; Morgan, D.; Szlufarska, I., J. Nucl. Mater., 414 (2011) 431439.

31. Motta, A. T., J. Nucl. Mater., 244 (1997) 227-250.

32. Hobbs, L. W.; Sreeram, A. N.; Jesurum, C. E.; Berger, B. A., Nucl. Instrum. Meth. B, 116 (1996) 18-25.

33. Wolf, D.; Okamoto, P. R.; Yip, S.; Lutsko, J. F.; Kluge, M., J. Mater. Res., 5 (1990) 286-301.

34. Luzzi, D. E.; Meshii, M., Journal of the Less Common Metals, 140 (1988) 193210.

35. Kulp, D. T.; Egami, T.; Luzzi, D. E.; Vitek, V., J. Non-Cryst. Solids, 156-158, Part 2 (1993) 510-513.

36. Hobbs, L. W., Nucl. Instrum. Methods Phys. Res., Sect. B, 91 (1994) 30-42.

37. Hobbs, L. W., J. Non-Cryst. Solids, 182 (1995) 27-39.

38. Novoa, J.; Braga, D.; Addadi, L.; Hobbs, L., Topological Approaches to the Structure of Crystalline and Amorphous Atom Assemblies. In Engineering of Crystalline Materials Properties, Springer Netherlands: 2008; pp 193-230.

39. Wang, S. X.; Wang, L. M.; Ewing, R. C.; Doremus, R. H., J. Non-Cryst. Solids, 238 (1998) 198-213.

40. Wang, S. X.; Wang, L. M.; Ewing, R. C.; Doremus, R. H., J. Non-Cryst. Solids, 238 (1998) 214-224.

41. Wang, S. X.; Lumpkin, G. R.; Wang, L. M.; Ewing, R. C., Nucl. Instrum. Meth. B, 166-167 (2000) 293-298.

42. Eby, R. K.; Ewing, R. C.; Birtcher, R. C., J. Mater. Res., 7 (1992) 3080-3102.

43. Naguib, H. M.; Kelly, R., Radiation Effects, 25 (1975) 1-12.

44. Devanathan, R.; Lam, N. Q.; Okamoto, P. R.; Meshii, M., Phys. Rev. B, 48 (1993) 42-51.

45. Xu, G. B.; Meshii, M.; Okamoto, P. R.; Rehn, L. E., J. Alloys Compd., 194 (1993) 401-405.

46. Trachenko, K., Journal of Physics-Condensed Matter, 16 (2004) R1491R1515.

47. Van Brutzel, L.; Crocombette, J. P., Nucl. Instr. and Meth. B, 255 (2007) 141145.

48. Weber, W. J., Nucl. Instrum. Methods Phys. Res., Sect. B, 166-167 (2000) 98106.

49. Pascucci, M. R.; Hutchison, J. L.; Hobbs, L. W., Radiation Effects, 74 (1983) 219-226.

50. Gibbons, J. F., Proceedings of the IEEE, 60 (1972) 1062-1096.

51. Wang, S. X.; Wang, L. M.; Ewing, R. C., Phys. Rev. B, 63 (2000) 024105.

52. Avrami, M., The Journal of Chemical Physics, 9 (1941) 177-184.

53. Nord, J.; Nordlund, K.; Keinonen, J., Phys. Rev. B, 65 (2002) 165329.

54. Nord, J.; Nordlund, K.; Keinonen, J., Phys. Rev. B, 68 (2003) 184104.

55. Hecking, N.; Heidemann, K. F.; Te Kaat, E., Nucl. Instrum. Methods Phys. Res., Sect. B, 15 (1986) 760-764.

56. $\quad$ Naguib, H. M.; Kelly, R., Radiat. Eff. Defect. S., 25 (1975) 1-12. 
57. Sickafus, K. E.; Valdez, J. A.; Williams, J. R.; Grimes, R. W.; Hawkins, H. T., Nucl. Instum. Meth. B, 191 (2002) 549-558.

58. Sickafus, K. E.; Minervini, L.; Grimes, R. W.; Valdez, J. A.; Hartmann, T., Radiat. Eff. Defect. S., 155 (2001) 133-137.

59. $\quad$ Sickafus, K. E.; Minervini, L.; Grimes, R. W.; Valdez, J. A.; Ishimaru, M.; Li, F.; McClellan, K. J.; Hartmann, T., Science, 289 (2000) 748-751.

60. Lian, J.; Zu, X. T.; Kutty, K. V. G.; Chen, J.; Wang, L. M.; Ewing, R. C., Phys. Rev. B, 66 (2002) 054108.

61. Devanathan, R.; Mitchell, J. N.; Sickafus, K. E.; Weber, W. J.; Nastasi, M., Mater. Sci. Eng., A, 253 (1998) 131-134.

62. Zallen, R., The Physics of Amorphous Solids. Wiley: New York, 1983. 